\title{
Multi-load Optimal Design of Burner-inner-liner Under Performance Index Constraint by Second-Order Polynomial Taylor Series Method
}

\author{
TU Gaoqiao, Wong Chun Nam, Zheng Min and Tang Kongzheng \\ Lanzhou University of Technology, School of Mechanical and Electronical Engineering, Lanzhou, Gansu 730050, China
}

\begin{abstract}
Using maximum expansion pressure of $\mathrm{n}$-decane, the aeroengine burner-inner-liner combustion pressure load is computed. Aerodynamic loads are obtained from internal gas pressure load and gas momentum. Multi-load second-order Taylor series equations are established using multi-variant polynomials and their sensitivities. Optimal designs are carried out using various performance index constraints. When 0.25 to 0.8 rectifications of different design variants are implemented, they converge under $5 \times 10^{-4} \mathrm{~d}$-norm difference ratio.
\end{abstract}

\section{Introduction}

According to the turbine combustion technique [1], maintenance costs on turbine and combustion chamber account for $60 \%$ of the whole aircraft maintenance. Therefore, high performance on the optimal design, operation reliability and structural safety are demanded on modern aeroengine thermal components. As burner inner liner (BIL) is the major component in combustion chamber, it becomes one of the most significant components of the aeroengine. BIL is a metallic thin-wall cylinder that controls the combustion, mixing and cooling processes. It guides the combustion chamber cylinder and rotors from thermal combustion products [2]. From the maintenance survey [3], BIL accounts for the $63 \%$ of the combustion chamber faults. Combustion chamber cylinder is $5 \%$ and fuel nozzle is $4 \%$. Since performance indices are determined by BIL mainly, it becomes the research focus on cannular combustion chamber design.

In the operation process of BIL, complicated pressure loads occurs which can be classified as follows: 1) Due to gas combustion, expansion pressure is exerted on its cylinder; 2) Pressure load is generated by the pressure different between the inner and outer cylinder surfaces. Most traditional optimization methods work on unique load where only one disciplinary analysis is involved. To improve the performance of BIL under these loads in different disciplinary, multi-load optimization technique is implemented in this design.

Multi-load optimization technique allows the multiple responses objective functions to be optimized simultaneously under the coupled design variants. This method caters for the complicated interactions among different disciplinary, and the requirements of various performance indices. Inequality constraints are imposed by these indices.

\section{Expansion pressure load created in fuel combustion}

The changes in reactant, product and composition mole fraction of $n$-decane and aviation fuel premixed combustion flame are basically consistent as indicated by Zeng[4]. Although the aviation fuel contains complicated ingredients, $n$-decane can be used for numerical simulation as alternative of aviation fuel. Details of his proposed reaction mechanism can describe detailed dynamic characteristics of $n$-decane premixed combustion. Because percentages of $\mathrm{C}$ and $\mathrm{H}$ within hexane hydrogen classes are the same, one can infer its complete combustion product proportions are the same. As a result, $n$-decane, propane and cyclohexane possess same maximum expansion pressure of $0.86 \mathrm{MPa}$.

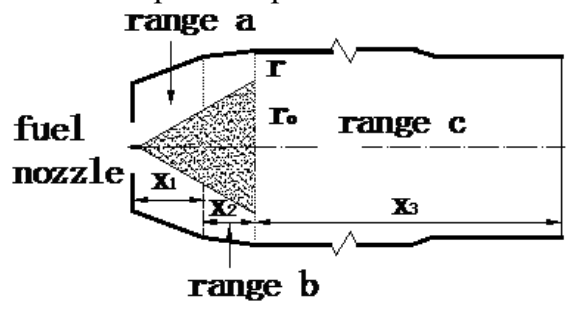

Figure 1. Sectional dimensions in each range.

From this known combustion pressure, BIL inside pressure distribution is obtained using inverse-square law $P_{0} / P_{1}=\left(r_{0}+r\right)^{2} / r_{0}^{2}$ as in Fig.1. In range a , $r_{0}+r=0.364 \cdot x_{1}+0.05=y_{1}$ for $0 \leq x_{1} \leq 0.055$.

In range $\mathrm{b}, \quad r_{0}+r=0.07002=y_{2} \quad$ for $0.055 \leq x_{2} \leq 0.095$. In range c, $r_{0}+r=\sqrt{\left(y_{2}-0.095 \tan (\pi / 6)\right)^{2}+\left(x_{3}-0.095\right)^{2}} /(0.07002-0.095 \tan (\pi / 6))$ 
for $0.095 \leq x_{3} \leq 0.51$. Using the distributed pressure on each range computed above, its thrust load is computed by equivalent nodal loads

$$
\begin{aligned}
F^{1} & =12 \cdot\left(F_{11} / 2+\sin (\alpha / 2+\pi / 4) \cdot F 0_{1}+\sin (\beta / 2+\pi / 4) \cdot F 0_{2}+\sin (\pi / 2-\beta / 2) \cdot F 0_{3}-\sin (\pi / 2-\gamma / 2) \cdot F 0_{8}-\sin (\gamma / 2+\pi / 2) \cdot F 0_{9}\right) \\
& =6.982 \times 10^{3} d a N
\end{aligned}
$$

where $\alpha, \beta, \gamma$ are the axial angles of ring $1,2,8$ respectively. $F_{n}$ is the pressure load on ring $n . F 0_{n}$ is the equivalent nodal load of ring $n$.

\section{Aerodynamic load generated by gas pressure and gas momentum}

According to the aerodynamic equation, when the gas passes the design model (Fig. 2), total thrust is the gas momentum difference between outlet and inlet. Set the gas pressure load inside as $P$. Taking the thrust direction as positive, aerodynamic force on BIL is $P-\hat{A_{0} P_{\text {in }}}+\hat{A_{1} P_{\text {out }}}$. Gas momentum is $G\left(V_{\text {out }}-V_{\text {in }}\right)$ with $G=50.7 \mathrm{~kg} / \mathrm{s}$. Thus we have the balance equation $P=G\left(V_{\text {out }}-V_{\text {in }}\right)+A_{0}^{\prime} P_{\text {in }}-A_{1}^{\prime} P_{\text {out }} \quad$ with $V_{\text {in }}=150 \mathrm{~m} / \mathrm{s}, V_{\text {out }}=600 \mathrm{~m} / \mathrm{s}$. Using the action and reaction rule, thrust load due to aerodynamic force is $F^{2}=-P$. Substituting all computed parts, one has

$$
F^{2}=G\left(V_{\text {in }}-V_{\text {out }}\right)-P_{\text {in }}\left(A_{0}+t\left(S_{11}+S_{3} \sin \beta\right)\right)+P_{\text {out }} A_{1}=3.7907 \times 10^{4} d a N
$$

where $t$ is the gas hole area, $S_{\mathrm{n}}$ is surface area of ring $n$. Therefore the total thrust load $F_{T}$ is composed of these multi-load as

$$
F_{T}=F^{1}+F^{2}=4.489 \times 10^{4} d a N
$$

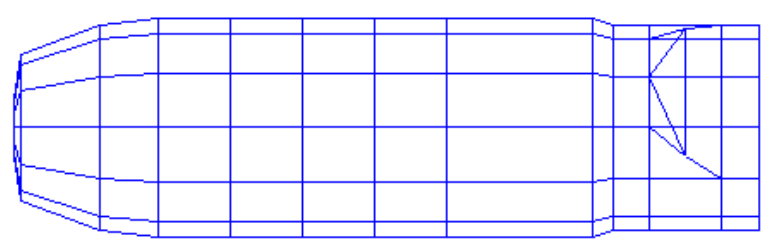

Figure 2. Design model of BIL.

\section{Establishment of multi-load optimization principle}

In this method, second-order Taylor Series expansion equation [5] is utilized to relate the changes of the multiload responses with the rectifications of design variants in the design model. By inverse computation, these rectifications in the design variants are estimated from the BFGS algorithm[6]. The optimization process is repeated until specific termination criteria are reached. Let
$\bar{Q}_{a}=\left(Q_{a 1}, Q_{a 2}, \cdots, Q_{a m}\right)^{T}$ be the $a$-th updated design variant vector, where $Q_{i}$ denotes the fuel nozzle jet angle, gas flow rate, fuel combustion pressure and compressor outlet pressure. Meanwhile $\bar{Q}_{d}=\left(Q_{d 1}, Q_{d 2}, \cdots, Q_{d m}\right)^{T}$ is the actual design variant vector. The selected sets of $n_{t}$ thrust response values $t^{k}$ and $n_{S}$ thrust response vectors $\bar{S}^{k}$ corresponding to the updated and actual structures are denoted by $\bar{Y}_{A}$ and $\bar{Y}_{D}$ respectively, where $\quad \bar{Y}_{A}=\left(t_{a}^{1}, t_{a}^{2}, \cdots, t_{a}^{n_{t}}, \bar{S}_{a}^{1}, \bar{S}_{a}^{2}, \cdots, \bar{S}_{a}^{n_{S}}\right)^{T} \quad$ and $\bar{Y}_{D}=\left(t_{d}^{1}, t_{d}^{2}, \cdots, t_{d}^{n_{t}}, \bar{S}_{d}^{1}, \bar{S}_{d}^{2}, \cdots, \bar{S}_{d}^{n_{S}}\right)^{T}$. In the thrust load system equation, $t^{k}$ are thrust force due to the fuel combustion, flow rate and air pressure. $\bar{S}^{k}$ are distributed stress due to expansion pressure and distributed air pressure on the cylinder surface.

In general, one can use second-order Taylor series expansion[7] to obtain the change of the $k$-th thrust response vector due to change in design variant $\delta Q_{i}$ $(i=1,2, \ldots, m)$ :

$$
\stackrel{-k}{S}_{d}-\stackrel{-k}{S}_{a}=\sum_{i=1}^{m} \frac{\partial \stackrel{-k}{S}_{a}}{\partial Q_{i}} \delta Q_{i}+\frac{1}{2 !} \sum_{i=1}^{m} \sum_{j=1}^{m} \frac{\partial^{2} \stackrel{-k}{S} Q_{i} \partial Q_{j}}{\partial Q_{i}} \delta Q_{j}+\stackrel{-k(2)}{\varepsilon_{t}}
$$

where $\bar{\varepsilon}_{S}^{k(2)}$ is the residual error vector in its expansion. For the $p$-th order expansion of the $k$-th thrust response value, one gets

$t_{d}^{k}-t_{a}^{k}=\sum_{i=1}^{m} \frac{\partial t_{a}^{k}}{\partial Q_{i}} \delta Q_{i}+\frac{1}{2 !} \sum_{i=1}^{m} \sum_{j=1}^{m} \frac{\partial^{2} t_{a}^{k}}{\partial Q_{i} \partial Q_{j}} \delta Q_{i} \delta Q_{j}+\varepsilon_{t}^{k(2)}$ in which $\varepsilon_{t}^{k(2)}$ is the residual error value in the expansion. Termination criterion is established to control the accuracy of iterative process. For the optimal design of BIL, the objective function is met when the sum of thrust response residual errors drop to the global minimum. As a result, the d-norm $\left\|\mathrm{d}_{a}\right\|$ difference ratio is chosen as the 
termination

indicator,

$\left\|\Delta \mathrm{d}_{a}\right\|=\left\|\mathrm{d}_{a}\right\|-\left\|\mathrm{d}_{a-1}\right\| /\left\|\mathrm{d}_{0}\right\|<5 \times 10^{-4}$ where

$\left\|\mathrm{d}_{a}\right\|=\sum_{k t}^{n_{t}} \sum_{k s=1}^{n_{s}}\left\|\left\{\bar{S}_{d}^{k s}-\bar{S}_{a}^{k s}, \frac{t_{d}^{k t}-t_{a}^{k t}}{t_{d}^{k t}}\right\}\right\|$.

$$
S_{a}^{-k}(\bar{Q})=\sum_{k 1=1}^{K 1} \sum_{k 2=1}^{K 2} \cdots \sum_{k m=1}^{K m} S_{a\langle k 1 k 2 \cdots k m\rangle}^{-k} L_{1}^{\langle k 1\rangle}\left(Q_{1}^{\langle k 1\rangle}\right) L_{1}^{\langle k 2\rangle}\left(Q_{2}^{\langle k 2\rangle}\right) \cdots L_{m}^{\langle k m\rangle}\left(Q_{m}^{\langle k m\rangle}\right)
$$

where $L_{i}^{k k i\rangle}\left(Q_{i}^{\langle k i\rangle}\right)$ is the Lagrange factor function of the $i$ th design variant at the kith interpolated stiffness value, given by

$$
\begin{aligned}
L_{i}^{\langle k i\rangle}\left(Q_{i}^{\langle k i\rangle}\right)= & \prod_{\substack{k=1 \\
k \neq k i}}^{k i}\left(\frac{Q_{i}-Q_{i}^{\langle k\rangle}}{Q_{i}^{\langle k i\rangle}-Q_{i}^{\langle k\rangle}}\right) \\
& t_{a}^{t}(\bar{Q})=\sum_{k 1=1}^{K 1} \sum_{k 2=1}^{K 2} \cdots \sum_{k m=1}^{K m} t_{a\langle k 1 k 2 \cdots k m\rangle}^{k} L_{1}^{\langle k 1\rangle}\left(Q_{1}^{\langle k 1\rangle}\right) L_{2}^{\langle k 2\rangle}\left(Q_{2}^{\langle k 2\rangle}\right) \cdots L_{m}^{\langle k m\rangle}\left(Q_{m}^{\langle k m\rangle}\right)
\end{aligned}
$$

First-order derivative terms can be obtained by direct derivative on Eqs. (7), (9) with respect to $Q_{i}$. For response vector and value derivatives [7] can be expressed as multiple variants rectification, the first-order thust

$$
\begin{aligned}
& \frac{\partial \bar{S}_{a}^{k}}{\partial Q_{i}}=\sum_{k 1=1}^{K} \sum_{k 2=1}^{K} \cdots \sum_{k m=1}^{K m} \bar{S}_{a\langle\langle k 1 k 1 \cdots m m\rangle}^{k} L_{1}^{\langle k 1\rangle}\left(Q_{1}^{\langle k 1\rangle}\right) \cdots \frac{\partial L_{i}^{\langle k\rangle}\left(Q_{i}^{\langle k i\rangle}\right)}{\partial Q_{i}} \cdots L_{m}^{\langle k m\rangle}\left(Q_{m}^{\langle k m\rangle}\right) \\
& \frac{\partial t_{a}^{k}}{\partial Q_{i}}=\sum_{k 1=1}^{K 1} \sum_{k 2=1}^{K 2} \cdots \sum_{k m}^{K m} t_{a\langle k 1 k 2 \cdots k m\rangle}^{k} L_{1}^{\langle k 1\rangle}\left(Q_{1}^{\langle k 1\rangle}\right) \cdots \frac{\partial L_{i}^{\langle k i\rangle}\left(Q_{i}^{\langle k i\rangle}\right)}{\partial Q_{i}} \cdots L_{m}^{\langle k m\rangle}\left(Q_{m}^{\langle k m\rangle}\right)
\end{aligned}
$$

where

$$
\frac{\partial L_{i}^{\langle k i\rangle}\left(Q_{i}^{\langle k i\rangle}\right)}{\partial Q_{i}}=\sum_{\substack{k=1 \\ k \neq k i}}^{K i} \frac{1}{Q_{i}^{\langle k i\rangle}-Q_{i}^{\langle k\rangle}} \prod_{\substack{k j=1 \\ k \neq k i k \\ k \neq \neq k}}^{K i}\left[\frac{Q_{i}-Q_{i}^{\langle k j\rangle}}{Q_{i}^{\langle k i\rangle}-Q_{i}^{\langle k j\rangle}}\right] .
$$

On the other hand, the second-order derivative consists of two terms namely the repeated differential and the unrepeated differential. For the unrepeated term, it is given by the direct first-order derivative of Eqs. 7,9 according to $Q_{i}$ involved. Special care is given to the repeated terms where direct second-order derivative with respect to $Q_{i}$ are encountered. For multiple variants, the second-order thust response vector and value derivatives [7] are expressd as

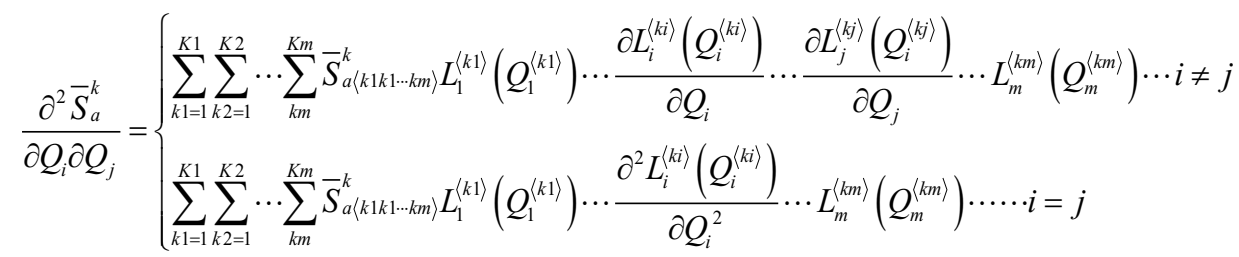

$$
\begin{aligned}
& \frac{\partial^{2} t_{a}^{k}}{\partial Q_{i} \partial Q_{j}}=\left\{\begin{array}{l}
\sum_{k 1=1}^{K 1} \sum_{k 2=1}^{K 2} \cdots \sum_{k m}^{K m} t_{a\langle k 1 k 2 \cdots k m)}^{k} L_{1}^{\langle k \mid\rangle}\left(Q_{1}^{\langle k \mid\rangle}\right) \cdots \frac{\partial L_{i}^{\langle k\rangle}\left(Q_{i}^{\langle k i}\right)}{\partial Q_{i}} \cdots \frac{\partial L_{j}^{\langle k\rangle}\left(Q_{j}^{\langle k\rangle}\right)}{\partial Q_{j}} \cdots L_{m}^{\langle k m\rangle}\left(Q_{m}^{\langle k m\rangle}\right) \cdots \cdots i \neq j \\
\sum_{k 1=1}^{K 1} \sum_{k 2=1}^{K 2} \cdots \sum_{k m}^{K m} t_{a\langle k 1 k 2 \cdots k m)}^{k} L_{1}^{\langle k \mid\rangle}\left(Q_{1}^{\langle k \mid\rangle}\right) \cdots \frac{\partial^{2} L_{i}^{\langle k\rangle}\left(Q_{i}^{\langle k\rangle}\right)}{\partial Q_{i}^{2}} \cdots L_{m}^{\langle k m\rangle}\left(Q_{m}^{\langle k m\rangle}\right) \cdots \cdots i=j
\end{array}\right.
\end{aligned}
$$

where 


$$
\frac{\partial^{2} L_{i}^{\langle k i\rangle}\left(Q_{i}^{\langle k i\rangle}\right)}{\partial Q_{i}^{2}}=\sum_{\substack{k=1 \\ k \neq k i}}^{K i} \frac{1}{Q_{i}^{\langle k i\rangle}-Q_{i}^{\langle k\rangle}} \sum_{\substack{k m=1 \\ k m \neq k i \\ k m \neq k}}^{K i} \frac{1}{Q_{i}^{\langle k i\rangle}-Q_{i}^{\langle k m\rangle}} \prod_{\substack{k j=1 \\ k j \neq k i \\ k j=k \\ k j \neq k m}}^{K i}\left[\frac{Q_{i}-Q_{i}^{\langle k j\rangle}}{Q_{i}^{\langle k i\rangle}-Q_{i}^{\langle k j\rangle}}\right]
$$

Substituting Eqs. (10), (12) into Eq. (4), second-order Taylor series expansion of thust response vector is furnished. Moreover, second-order thust response value equation of Eq. (5) is completed by Eqs. (11), (13).

\section{Normalization of taylor series equation using accuracy number}

Here we carry out the normalization of the optimization equations. For the response vectors, we reduce the data precision as small as possible, while not affecting its variation characteristics. We multiply the equations in each discipline by accuracy numbers, so that they are normalized to the same precision level. In this case, the computation problems such as the singularity, accuracy and convergency can be avoided. For example, accuracies of the distributed stress and pressure are $10^{19}$ and $10^{6}$ respectively, then the normalization accuracy numbers are $\left[10^{-19}, 10^{-6}\right]$. Under this treatment, faster and more accurate convergences are attained.

\section{Optimal design of BIL under performance index constraint}

In this optimization approach, inequality constraints are imposed using various performance indices. Specific thrust is constrained in the range $60 \leq F_{\mathrm{s}}=F_{T} / G \leq 80 \mathrm{da} N \cdot \mathrm{s} / \mathrm{kg} \quad ; \quad$ specific fuel consumption

is

$0.8 \leq C_{F}=3600 \mathrm{f} / F_{S} \leq 1.0 \mathrm{~kg} /(\mathrm{h} \cdot \mathrm{daN}) \quad$ where $f \approx 0.018 \quad ; \quad$ thrust weight ratio is $3.5 \leq F_{w}=F_{T} / W_{g} \leq 4.0$ and compressor outlet pressure ratio is $1.15 \leq P_{r}=P_{\text {out }} / P_{\text {atm }} \leq 1.35$ where $P_{a t m}$ is the atmospheric pressure.

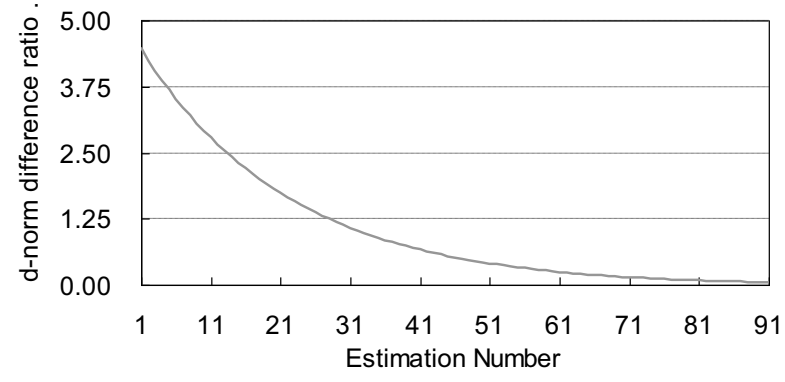

Figure 3. Convergence of $\left\|\Delta \mathrm{d}_{a}\right\|$ under 0.5 rectification of $Q_{2}$ and $Q_{4}$.

Using different combinations of one to four $Q_{i}$, various 0.25 to 0.8 rectification cases are established.
When two design variants, gas flow rate $Q_{2}$ and compressor outlet pressure $Q_{4}$ are rectified in 0.5 level, their optimization and convergence processes are recorded as follows.

From Fig. 3, the minimization of $\left\|\Delta \mathrm{d}_{a}\right\|$ is faster up to estimation 40. It becomes slower up to estimation 80 , and stable afterwards. Termination criterion is met at estimation 91 where the $\left\|\Delta \mathrm{d}_{a}\right\|$ drops to $4.8 \times 10^{-4}$.

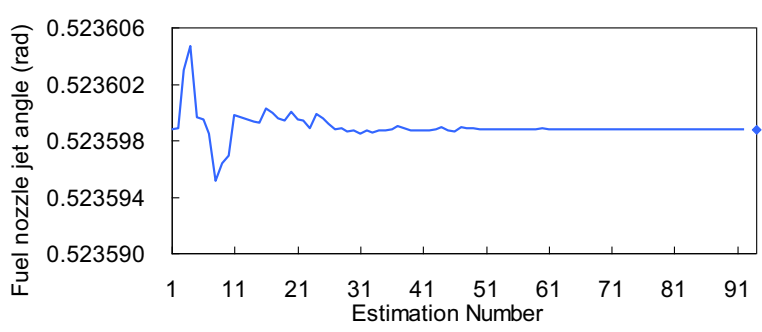

Figure 4. Estimation pattern of $Q_{1}$ under 0.5 rectification

From Fig. 4, rapid adjustment of $Q_{1}$ occurs up to estimation 40. Then it becomes stable afterwards. As its rectification is relatively small, wave-like pattern having peak and valley is observed.

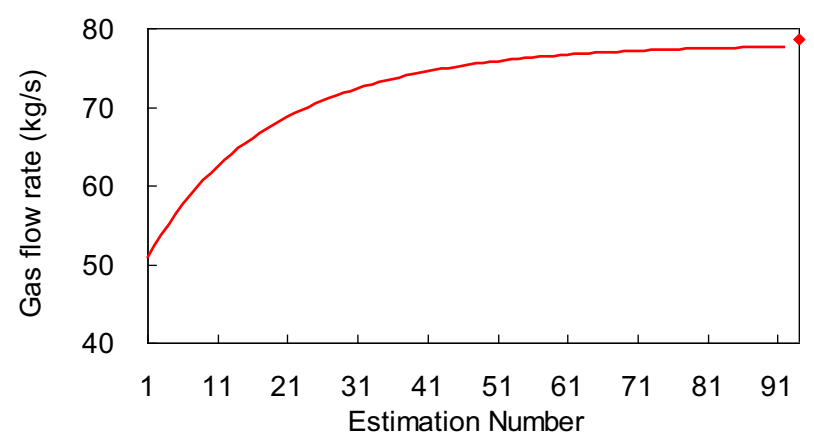

Figure 5. Estimation pattern of $Q_{2}$ under 0.5 rectification.

Estimation process of $Q_{2}$ is illustrated in Fig.5. Similar to the pattern of $\|\mathrm{d}\|$, it increases rapidly up to estimation 40. Afterwards, it changes gradually until termination criterion is met at estimation 91 where its percentage error is constrained by the performance indices at $1.21 \%$. From the estimation pattern of $Q_{3}$, its rapid adjustment occurs up to estimation 30. Then it becomes stable afterwards. Not the same as $Q_{1}$, its rectification cycle is shorter leading to less significant peak and valley features.

Clear cut pattern is observed as in Fig. 6. As its nominal value is relatively large among the other variants, its priority is high in the pattern. Thus its optimization is 
rapid and accurate. Using 0.25 rectification for same design variants, 92 estimations are needed. Thus, the convergence rate is slightly decreased for smaller rectification level.

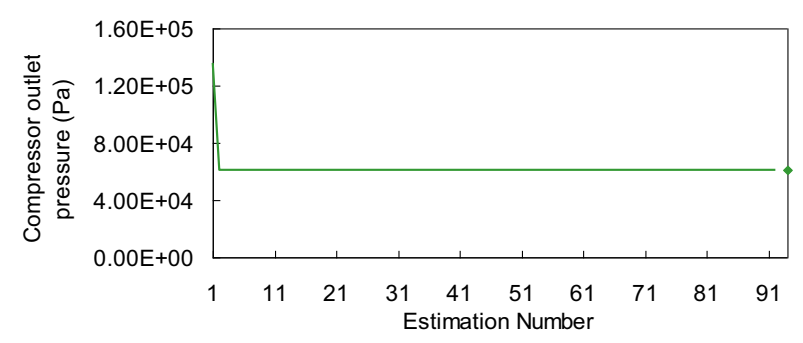

Figure 6. Estimation pattern of $Q_{4}$ under 0.5 rectification.

When three design variants $Q_{1}, Q_{2}, Q_{4}(m=3)$ are rectified in 0.8 level, their optimization and convergence processes are recorded as follows.

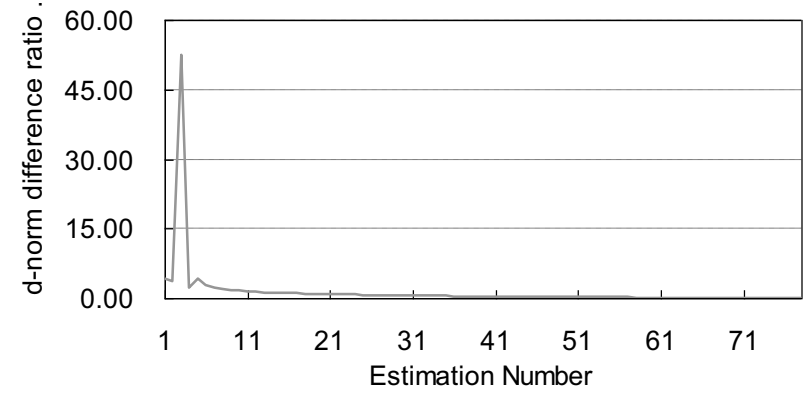

Figure 7. Convergence of $\left\|\Delta \mathrm{d}_{a}\right\|$ under 0.8 rectification of $Q_{1}, Q_{2}, Q_{4}$.

From Fig. 7, $\left\|\Delta \mathrm{d}_{a}\right\|$ is ramped up to the peak 52.5 at estimation 3. Then it drops rapidly to 2.26 and minimizes gradually afterwards. Termination criterion is met at estimation 78 where $\left\|\Delta \mathrm{d}_{a}\right\|$ drops to $4.8 \times 10^{-4}$.

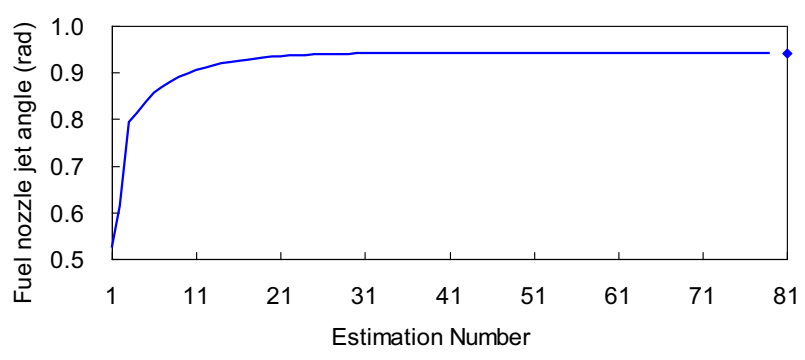

Figure 8. Estimation pattern of $Q_{1}$ under 0.8 rectification.

Estimation process of $Q_{1}$ is illustrated in Fig. 8 . Initially, it ramps up rapidly to $7.93 \mathrm{rad}$ at estimation 3 . Afterwards, it increases gradually to the +0.8 rectification level with $1.47 \times 10^{-4} \%$ deviation.

Estimation process of $Q_{2}$ is illustrated in Fig. 9. It increases from estimation 3 gradually near +0.8 rectification level of $89.4 \mathrm{~kg} / \mathrm{s}$ where it is constrained by the performance indices at $2.08 \%$. As $Q_{3}$ rectification is zero, wave-like pattern having large peak and valley of amplitudes up to $7.5 \times 10^{6} \mathrm{~Pa}$ is observed in the initial stage. Then it drops gradually to the initial level with $-6.94 \times 10^{-4} \%$ deviation.

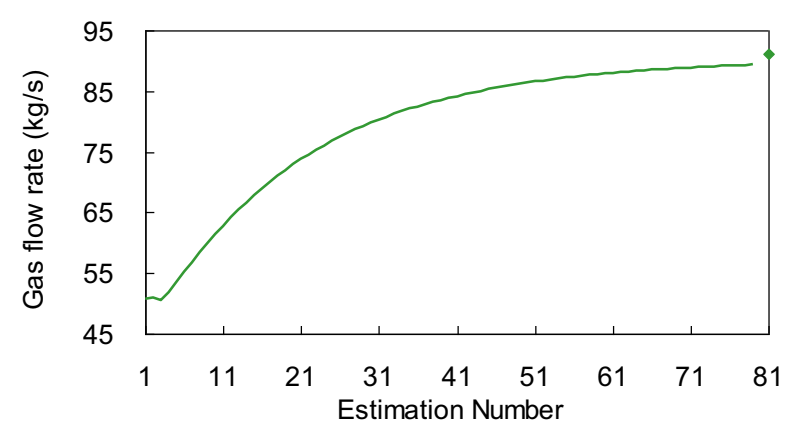

Figure 9. Estimation pattern of $Q_{2}$ under 0.8 rectification.

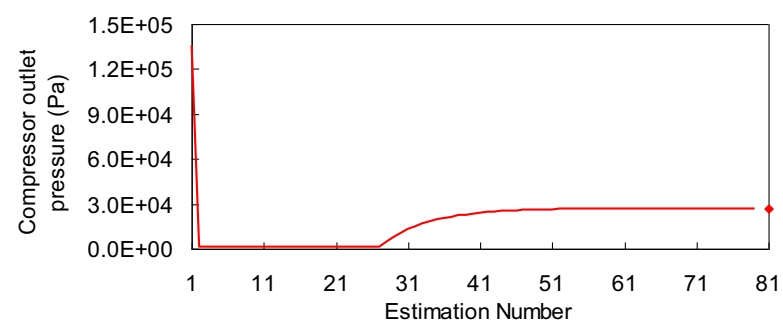

Figure 10. Estimation pattern of $Q_{4}$ under 0.8 rectification.

From Fig. $10 Q_{4}$ drops rapidly from $1.36 \times 10^{5} \mathrm{~Pa}$ to minimum level of $1.36 \times 10^{3}$. It remains stationary until estimation 27, and increases gradually to the -0.8 rectification level with $4.44 \times 10^{-2} \%$ deviation. Using same design variants with 0.25 rectification, 87 estimations are required. Therefore, the convergence rate is lower for larger rectification level. When $m=1,97$ estimations are needed in both 0.25 and 0.8 rectification cases. For $m=4$, there is 69 estimations in 0.25 rectification case. In the 0.8 rectification, 44 estimations are required. When rectification level is larger, convergence rate is decreased. Meanwhile, for the increase in $m$, the convergence rate is increased. For the illustrated cases, $m=2,3$, their convergence rates follow these trends.

\section{Summary}

BIL combustion pressure load $F^{1}$ is calculated to be $6.982 \times 10^{3} d a N$ using $0.86 \mathrm{MPa} n$-decane expansion pressure. Aerodynamic load $F^{2}$ is obtained as $3.7907 \times 10^{4} d a N$. Using developed equations, 0.25 to 0.8 rectifications of one to four design variants constrained by various performance indices converge under $5 \times 10^{-4}\left\|\Delta \mathrm{d}_{a}\right\|$ criterion. In general, larger rectification level leads to slower convergence. This trend is more significant for larger $m$. When $m$ increases, its convergence becomes faster. 


\section{Acknowledgements}

This research is supported by the National Natural Science Foundation of China under grant numbers 50905028 and 51275554.

\section{References}

1. X. Hou, H. Ji, Q. Liu, et al., High performance aviation gas turbine combustion technique. Peking: Militrary Defence Industrial Press, (2002), pp. 5-11.

2. Y. Wang, Aeroengine Principle. Peking: Beihang University Press, (2009), pp. 116-124.

3. S. Peng, Aeroengine Combustion Chamber Structure. Peking: Militrary Defence Industrial Press, (1978), pp. 3-7.

4. W. Zeng, J. Liu, X. Chen, M. Jie, Detailed reaction kinetic modeling of $n$-decane premixed combustion,
Journal of Aerospace Power, 26(10):2258-2266. (2011)

5. C.N. Wong, A.A. Barhorst, Polynomial interpolated Taylor series method for parameter identification of nonlinear dynamic system, ASME Journal of Computational and Nonlinear Dynamics, 1(3):248256 (2006).

6. C.N. Wong, J. Xiong, H. Huang, T. Hu, Damage Detection of Space Truss using Second Order Polynomial Method with BFGS Quasi-Newton Optimization, Proceedings of the ASME 2010 IDETC/CIE, DETC2010-28091, (2010), pp.753-762.

7. C.N. Wong, J. Xiong, H. Huang, Y.J. Zhao, A polynomial algorithm for model updating of engineering truss, Mechanics Based Design of Structures and Machines, 38:1-24, (2010). 